\title{
Estudio de percepción de pescadores artesanales sobre aspectos de conservación marina y áreas marinas protegidas en la región de Magallanes
}

\author{
Study of artisanal fishermen perception on marine \\ conservation and marine protected areas in Magallanes region
}

Belén Guarda ${ }^{1}$ \& Alejandro Vila ${ }^{1}$

\section{Resumen}

La conservación marina es un desafío complejo y su éxito está generalmente determinado por factores sociales. Consecuentemente, antes de promover la creación de áreas marinas protegidas (AMP), resulta necesario contemplar cuál es la visión de los diferentes actores sociales sobre las mismas, sus percepciones, intereses y expectativas, para minimizar conflictos y lograr el apoyo y la aceptación necesarios para su implementación y manejo efectivo. En este contexto, y en una etapa previa a la creación de un Área Marina Costera Protegida de Múltiples Usos (AMCP-MU) en el Seno Almirantazgo, evaluamos la percepción de pescadores artesanales de la región de Magallanes sobre la conservación de la biodiversidad, las AMP y el proyecto de creación de una nueva AMCP-MU. Se entrevistaron 35 pescadores en caletas de las ciudades de Porvenir, Punta Arenas y Puerto Natales. Los entrevistados demostraron conocimientos elementales sobre las temáticas tratadas que, en general, fueron obtenidas a través de diferentes fuentes y medios. El 43\% de los entrevistados percibió a las AMP como zonas cerradas, destinadas a proteger especies y en donde las actividades humanas representan una amenaza para estas, mientras que un $23 \%$ indicó que no se trata de zonas necesariamente cerradas y que permiten la realización de ciertas actividades. Respecto a la creación de una AMCP-MU en el Seno Almirantazgo, el $68 \%$ de ellos mencionó que no debiera generar restricciones para el desarrollo de sus actividades, como así tampoco para las de otros actores (e.g. sector turístico). En cuanto a la percepción sobre los actores vinculados con la conservación de la biodiversidad en la región, un $54 \%$ de los pescadores mostró cierta aprensión frente a los servicios públicos, por su rol de regulación y fiscalización, mientras que el sector del turismo y de las organizaciones no gubernamentales fue visualizado más positivamente. Los resultados de este trabajo contribuyen al entendimiento de la dimensión humana de la conservación marina en Magallanes, resaltan la importancia de la comunicación para encontrar una base común de contacto entre actores con intereses y experiencias culturales diferentes, y brindan antecedentes para el proceso de creación e implementación de AMP.

\section{Palabras clave:}

Patagonia, Seno Almirantazgo, pesca, ONG, turismo
Abstract

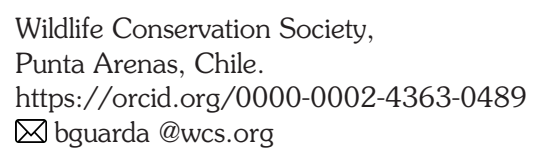

Marine conservation is a complex challenge and its success is generally determined by social factors. Consequently, before promoting the creation of marine protected areas (MPAs), it is necessary to consider the stakeholder's vision upon them, their perceptions, interests and expectations, in order to minimize conflicts and achieve the necessary support for their implementation and effective management. In this context, and considering the project to propose the creation 
of a Multiple-Use Coastal Marine Protected Area (AMCP-MU) in the Almirantazgo Sound, we evaluated the perception of artisanal fishermen from the Magallanes region on the topics of biodiversity conservation, MPAs and the project to create a new AMCP-MU. Thirty-five fishermen were interviewed in different coves from Porvenir, Punta Arenas and Puerto Natales cities. In general, the interviewees demonstrated basic knowledge about the aforementioned topics, which, in general, was obtained through different means. Forty three percent of the fishermen interviewed, perceived MPAs as closed areas, intended to protect species and were human activities represent a threat to them, while $23 \%$ indicated that MPAs are not necessarily closed areas, allowing the development of some activities. Regarding the creation of an AMCP-MU in the Almirantazgo Sound, $68 \%$ of the fishermen stated that it should not generate restrictions for the development of their activities, nor for those of other sectors (e.g. tourism sector). In regards of the fishermen's perception upon the stakeholders linked to biodiversity conservation in the region, $54 \%$ of the fishermen showed some apprehension towards public services, due to their regulating and supervising role, while the tourism sector and Non-governmental organization were viewed more positively. The results of this work contribute to the understanding of the human dimension of marine conservation in Magallanes, highlight the importance of communication to find a common ground between diverse actors with different cultural interests and experiences, and provide contextual background for the process of creation and implementation of MPAs.

\section{Key words:}

Patagonia, Seno Almirantazgo, fishing, NGO, tourism

\section{INTRODUCCIÓN}

Los océanos juegan un rol clave en la provisión de funciones y servicios ecosistémicos a nivel global (Palumbi et al. 2009; Townsend et al. 2018). Sin embargo, enfrentan fuertes presiones antrópicas que atentan contra su salud (Poloczanska et al. 2016; Kroodsma et al. 2018; Di Minim et al. 2019). La sobrepesca, la destrucción de hábitats, los efectos del cambio climático y la contaminación, entre otras presiones, amenazan tanto a la biodiversidad marina como a los beneficios que brinda a la sociedad (Worm et al. 2006, 2007; McCauley et al. 2015). En este contexto, las áreas marinas protegidas (AMP) han surgido como una herramienta muy importante para enfrentar estas amenazas, pues permiten proteger hábitats; funcionalidad, estructura e integridad ecosistémica; diversidad, riqueza, tamaño y densidad de especies (Edgar et al. 2007; Lester et al. 2009; Edgar et al. 2014).

Considerando tanto las tendencias en el deterioro de los ecosistemas marinos como los efectos de las AMP en la conservación de la biodiversidad y el uso sustentable de los recursos marinos, la comunidad internacional se propuso proteger el $10 \%$ de las regiones marinas, a través del Plan Estratégico para la Diversidad Biológica 2011-2020, la Meta 11 de Aichi (Convention on Biological Diversity, 2010) y el objetivo 14 de Desarrollo Sustentable de las Naciones Unidas (UN, 2015). En el 2016, la Resolución 050-SP del Congreso Mundial de la Naturaleza alienta a los miembros de la Unión Internacional para la Conservación de la Naturaleza a designar, e implementar efectivamente, al menos un $30 \%$ de cada hábitat marino en una red de AMP y otras medidas de conservación eficaces (UICN, 2016).

Estas iniciativas han promovido una amplia expansión de la cobertura de AMP a nivel global, que alcanzó cerca de 27 millones de $\mathrm{km}^{2}$ (7,43\% de la superficie marina) hacia el 2020 (UNEP-WCMC \& IUCN, 2020). Chile se ha sumado a este compromiso $y$, entre los años 2015 y 2020, alcanzó un nivel significativo de progreso en la expansión de la superficie de AMP, que actualmente cubre alrededor de un $41,2 \%$ de su Zona Económica Exclusiva (UNEP-WCMC \& IUCN, 2020). Sin embargo, el establecimiento de AMP en el país se ha caracterizado por deficiencias en cuanto al diseño, pues en general respondió a una lógica de oportunidad y priorización de sitios aislados que presentaban bajo grado de conflictividad (Fernández \& Castilla, 2005; Hucke-Gaete et al. 2010; Kuempel et al. 2019), como así también por la falta de evaluación previa del grado de aceptación de la creación de AMP por parte de la comunidad (Zorondo-Rodríguez et al. 2019). 
La costa chilena Patagónica es una de las regiones de fiordos más extensas a nivel global (Pantoja et al. 2011), sustenta una alta diversidad de invertebrados (Häussermann \& Försterra, 2009) y sitios de reproducción y alimentación de vertebrados (Aguayo-Lobo et al. 2007; Falabella et al. 2009; Hucke-Gaete et al. 2010). Tanto por su diversidad de especies como productividad, resulta relevante por los bienes y servicios que provee a la sociedad (Iriarte et al. 2010; Hucke-Gaete et al. 2010; Outeiro et al. 2015; Nahuelhual et al. 2019), en particular como sustento de algunas de las principales actividades socioeconómicas de la región, como la pesca artesanal, que se encuentra posicionada en tercer lugar a nivel nacional en términos de inscripciones de empleados en el rubro (Servicio Nacional de Pesca y Acuicultura, 2019), y el turismo de intereses especiales, que está incrementando en forma sostenida (Cáceres et al. 2015).

En particular, la pesca artesanal está basada en la extracción de moluscos, equinodermos, peces y crustáceos (Guzmán et al. 2010). En el año 2019, la región aportó con más de 31.800 toneladas de recursos marinos a los desembarques nacionales, operando con 792 embarcaciones y empleando a más de 6200 pescadores (Vila et al. 2017; Servicio Nacional de Pesca y Acuicultura, 2019). Esta actividad se desarrolla bajo condiciones climáticas adversas y, generalmente, en puntos de extracción que se encuentran en zonas aisladas, lo cual implica movimientos de más de 18 horas de navegación y el desarrollo de actividades o movimientos dentro de las AMP de la región. Consecuentemente, la percepción de los pescadores artesanales frente a las AMCP-MU existentes (e.g. Francisco Coloane), como así también ante la creación de nuevas AMP es relevante.

En la región patagónica existen 12 áreas destinadas a la protección de espacios marinos, además de un parque marino y seis parques $y$ reservas nacionales que incluyen extensas porciones de mar dentro de sus límites (Ministerio del Medio Ambiente, 2020). Entre estas, las áreas marino costeras protegidas de múltiples usos (AMCP-MU) son consideradas una herramienta fundamental para el manejo integrado de los recursos marinos y la promoción del uso sustentable de los mismos, como así también para la protección, restauración y conservación de la biodiversidad y la cultura de un área (Montiel \& Jara, 2020). Dado que en las
AMCP-MU pueden coexistir diferentes actividades $e$ interactuar distintos actores con los objetos de conservación que se pretenden proteger en ellas (Dudley, 2008), resulta relevante considerar aspectos de la dimensión humana de la conservación para compatibilizar objetivos biológicos y sociales que podrían resultar contradictorios y generar conflictos (Christie et al. 2003; Pomeroy et al. 2007 a,b; Charles \& Wilson, 2009).

A pesar de la relevancia y necesidad de considerar aspectos sociales tanto en las propuestas para la creación de AMP como en su diseño e implementación, la mayoría de los estudios sobre las percepciones, intereses y expectativas de los actores sociales locales frente a la creación de un área protegida han sido realizados después de haber sido creadas (Lopes et al. 2013; Bennett \& Dearden, 2014; Brain et al. 2020). Sin embargo, los estudios de percepción previos a la creación de un AMP demuestran la relevancia de considerar la participación temprana de los usuarios en el proceso de planificación y diseño del área para reducir conflictos, lograr apoyo y aceptación para la creación, y establecer las bases para su implementación y manejo efectivo (Christie et al. 2003; Pomeroy et al. 2007b; Charles \& Wilson, 2009; Heck \& Dearden, 2012; Cárcamo et al. 2014; Zorondo-Rodríguez et al. 2019).

El objetivo de nuestro trabajo fue evaluar las percepciones de los pescadores artesanales de la región de Magallanes con relación a la biodiversidad, las AMP, los actores que están involucrados con el manejo de estas y la potencial creación de un AMCP-MU en Seno Almirantazgo, antes de su declaratoria formal. Nuestros resultados resultan relevantes para los procesos de declaratoria de AMP y son también un insumo clave para abordar la planificación de procesos participativos que contribuyan tanto con el diseño como la implementación de AMP.

\section{MATERIALES Y MÉTODOS}

\section{Características del área de estudio}

Este estudio se realizó en diferentes localidades de la región de Magallanes entre octubre de 2014 y febrero de 2015, en los principales terminales $\mathrm{y} / \mathrm{o}$ caletas pesqueras 
artesanales que congregan una alta concurrencia de pescadores. En particular, se trabajó en caletas de Punta Arenas (Barranco Amarillo, bahía Punta Carrera y bahía Mansa), Puerto Natales (terminal pesquero) y Porvenir (bahía Chilota) (Fig.1).

El área de estudio involucra el maritorio y las AMP de la región. Asimismo, se consideró la existencia de una propuesta para declarar un AMCP-MU en el Seno Almirantazgo (Vila et al. 2017). Esta área es relevante para la pesca del Ostión del Sur (Chlamys vitrea) y otras especies (Guzmán et al. 2010; Subsecretaría de Pesca y Acuicultura, 2010; Vila et al. 2017), y también se destaca por su valor para la conservación en términos ecológicos, turísticos e históricoculturales (Fernández, 2014, Brain et al. 2020). En este contexto, el estudio se realizó en las etapas previas a la declaración del Seno Almirantazgo como AMCP-MU, que fue formalmente creada el 20 de julio de 2018 mediante el Decreto Supremo $\mathrm{N}^{\circ} 11$ del Ministerio del Medio Ambiente.

\section{Enfoque metodológico}

Se realizaron entrevistas semiestructuradas basadas en preguntas generales para orientar la conversación sobre la biodiversidad y las áreas protegidas de la región de forma indirecta, compartiendo parte de la jornada trabajo y los momentos de descanso de los entrevistados. De esta forma, se favoreció la espontaneidad, se evitó influenciar el discurso de los participantes de las entrevistas y se conoció en forma más directa su realidad

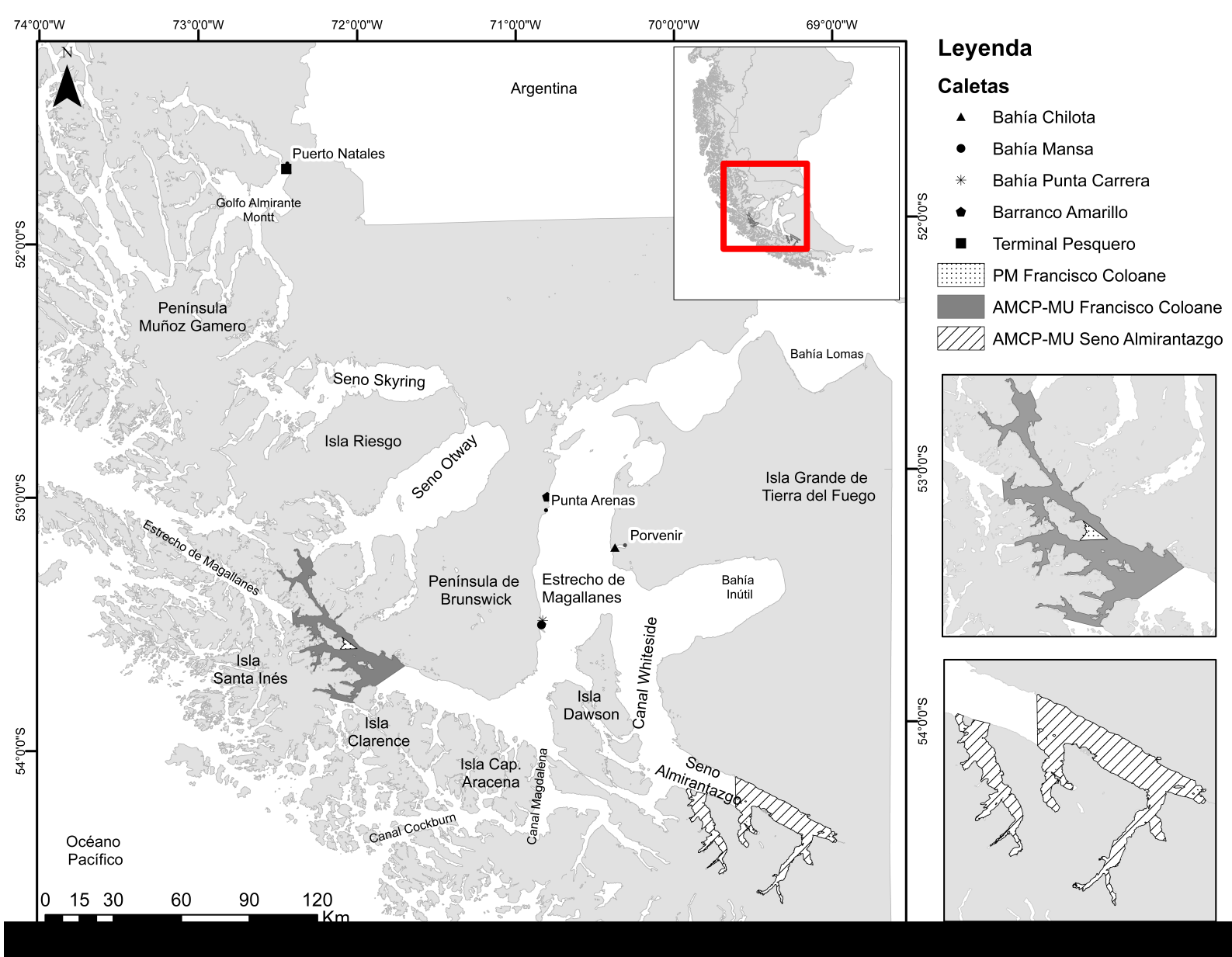

Fig. 1. Localidades en donde se realizaron las entrevistas a los pescadores artesanales y ubicación de las AMCP-MU mencionadas en este estudio, región de Magallanes y la Antártica Chilena. 
Tabla 1. Criterios de clasificación del nivel de conocimiento de los entrevistados en relación a los temas abordados durante las entrevistas.

Nivel de conocimiento
Criterio de clasificación

\begin{tabular}{ll}
\hline Bajo & $\begin{array}{l}\text { No tiene conocimiento sobre el tema abordado o el entendimiento del mismo es muy bajo como para tener } \\
\text { una opinión o percepción clara. }\end{array}$ \\
\hline Medio & $\begin{array}{l}\text { Muestra un conocimiento básico sobre la temática tratada. No maneja mucha información sobre las leyes } \\
\text { y los reglamentos que rigen su actividad y las áreas marinas protegidas (AMP). }\end{array}$ \\
\hline Alto & $\begin{array}{l}\text { Cuenta con un conocimiento preciso y claro de la temática tratada. Muestra conocimiento sobre la } \\
\text { legislación y reglamentación vigente sobre su actividad y las AMP. }\end{array}$
\end{tabular}

(Agrech, 2015). En particular, se abordaron aspectos vinculados con la biodiversidad de la región, las diferentes figuras de protección, las áreas protegidas existentes, qué tipos de actividades permiten y de qué forma afectan las actividades de los encuestados, la percepción frente a la creación de una nueva AMP y sobre los actores vinculados con la conservación de la biodiversidad en Magallanes.

Las entrevistas se realizaron al azar y sin haber tomado contacto previo con los entrevistados. Estas mismas siempre fueron realizadas por la misma persona y grabadas con el consentimiento de los presentes $y$, complementariamente, se tomaron notas y observaciones sobre la conversación. Los detalles sobre este estudio fueron compartidos, gradualmente, a lo largo del encuentro o al final del mismo. Posteriormente, las entrevistas fueron transcritas para sistematizar la información obtenida sobre cada temática y, en algunos casos, categorizar las respuestas según el nivel de conocimiento demostrado por los entrevistados (Tabla 1).

Se entrevistaron 35 pescadores artesanales con más de 15 años de trayectoria en la actividad, en las caletas donde se realizan los mayores desembarques de recursos pesqueros provenientes de la porción sur de Tierra de Fuego (Tabla 2). Si bien se estima que desde las caletas visitadas operan unas 500 embarcaciones (lo que equivale a la misma cantidad de capitanes por embarcación), el número de entrevistados estuvo condicionado por la cantidad de pescadores presente en estas y/o con su disponibilidad para ser encuestados. La mayoría (66\%) de los entrevistados utilizaba la caleta de Barranco Amarillo y el Seno Almirantazgo (77\%) durante sus faenas pesqueras al momento del estudio (Tabla 2).

Tabla 2. Caletas en donde se realizaron entrevistas y número de pescadores artesanales entrevistados en cada una de ellas, en la región de Magallanes.

\begin{tabular}{|c|c|c|}
\hline Nombre de caleta & Comuna / Capital provincial & $\begin{array}{l}\mathrm{N}^{\circ} \text { de pescadores } \\
\text { artesanales entrevistados }\end{array}$ \\
\hline Barranco Amarillo (5305’46,6”S 7052’29,0”O) & Punta Arenas & 23 \\
\hline Bahía Mansa (53³6’26,6”S 7055’59,9”O) & Punta Arenas & 2 \\
\hline Punta Carrera (53⒊'57,4”S 7056’00,0”O) & Punta Arenas & 1 \\
\hline 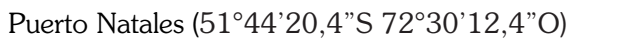 & Puerto Natales & 5 \\
\hline Bahía Chilota (53¹8’14,0”S 70²6’09,7”O) & Porvenir & 4 \\
\hline \multicolumn{3}{|l|}{ Usuario directo del Seno Almirantazgo } \\
\hline Sí & & 27 \\
\hline No & & 8 \\
\hline
\end{tabular}




\section{RESULTADOS}

Conocimientos sobre conservación de la biodiversidad y áreas protegidas

El tipo de respuesta no fue homogéneo entre los niveles de conocimiento evaluados en los entrevistados. Los participantes de las entrevistas mostraron conocimientos intermedios, sobre conservación y biodiversidad (66\%), formas de hacer conservación y áreas protegidas (49\%) y áreas protegidas existentes en la región (60\%) (Tabla 3). Por ejemplo, reconocieron la existencia del AMCP-MU Francisco Coloane, a la cual mencionaron como Carlos III, como un área de conservación importante para la protección de la ballena jorobada y el desarrollo de actividades turísticas.

Respecto al conocimiento adquirido por los participantes consultados sobre las temáticas tratadas, señalaron que fue obtenido por interés personal, como producto de su trabajo en terreno, a través del encuentro con embarcaciones dedicadas al turismo o la exploración, el encuentro con especies protegidas o con los científicos que las investigan. Otras fuentes importantes de información para adquirir conocimientos sobre las áreas protegidas estuvieron ligadas con la legislación existente sobre los recursos marinos, el relacionamiento regular con personas del ámbito científico, la participación en talleres $y$ reuniones con autoridades y organizaciones de conservación, y a través de los medios de comunicación.
Percepciones sobre las áreas marinas protegidas

Se identificaron cinco tipos de percepciones sobre qué es un área protegida (Fig. 2A). Para el $43 \%$ de los entrevistados se trata de una zona cerrada, destinada a proteger especies y en donde las actividades humanas representan una amenaza directa para estas y, por lo tanto, se encuentran prohibidas. No obstante, la percepción de un $23 \%$ de los pescadores consultados indicó que son zonas no necesariamente cerradas y permiten la realización de actividades (e.g. turismo y pesca artesanal). En particular, los entrevistados destacaron que son áreas que están destinadas a proteger espacios y especies, asociadas al manejo de los recursos pesqueros y son administradas por las autoridades públicas, o que están vinculadas a la actividad turística (Fig. 2A). Finalmente, alrededor de un $10 \%$ de los participantes de las entrevistas desconocía cuál es el alcance de un área protegida.

En concordancia con la visión de que las áreas protegidas son zonas cerradas al uso, el $57 \%$ de los entrevistados las percibieron como una amenaza para sus actividades (Fig. 2B), pues entendían que pueden limitarlas o prohibirlas. Sin embargo, los pescadores restantes manifestaron que la actividad pesquera no se vería afectada por la presencia de áreas protegidas $e$, incluso, podría ser beneficiada por estas (Fig. 2B).

Percepciones acerca del proyecto de creación de un AMCP-MU en el Seno Almirantazgo

La percepción más frecuente $(68 \%)$ de los entrevistados frente a la creación de una AMCP-

Tabla 3. Nivel de conocimiento de los pescadores artesanales de Magallanes entrevistados en relación a temáticas de conservación, biodiversidad y áreas protegidas.

\begin{tabular}{|c|c|c|c|}
\hline Temática tratada & Niveles de conocimiento & $\mathrm{N}^{\circ}$ de entrevistados & $\%$ \\
\hline \multirow{3}{*}{ Conservación y biodiversidad } & Ninguno o poco & 6 & 17 \\
\hline & Medio & 23 & 66 \\
\hline & Alto & 6 & 17 \\
\hline \multirow{3}{*}{ Formas de hacer conservación y áreas protegidas } & Ninguno o poco & 13 & 37 \\
\hline & Medio & 17 & 49 \\
\hline & Alto & 5 & 14 \\
\hline \multirow{3}{*}{$\begin{array}{l}\text { Áreas protegidas existentes en la región (Parques y } \\
\text { AMCP-MU) }\end{array}$} & Ninguno o poco & 7 & 20 \\
\hline & Medio & 21 & 60 \\
\hline & Alto & 7 & 20 \\
\hline
\end{tabular}


Es una zona de protección o manejo de los recursos, abierta a la actividad

Es una zona relacionada al turismo

Es una zona de conservación de biodiversidad, no necesariamente cerrada

Es una zona cerrada (excluye actividades económicas)

No tiene una opinión formada

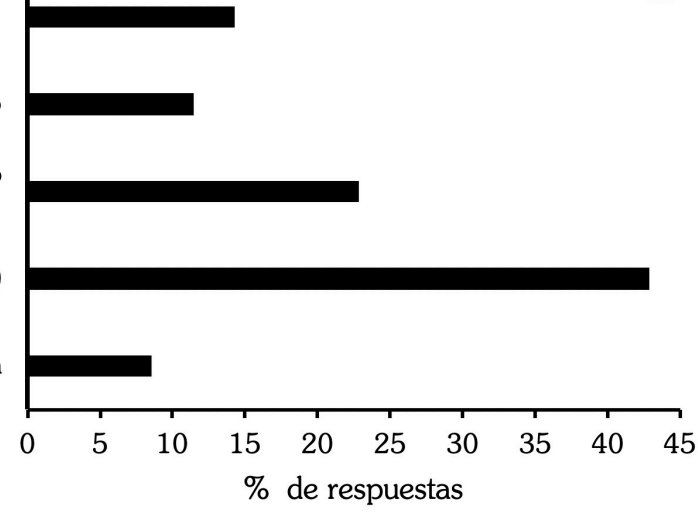

A

B

Es una amenaza para su actividad

No influencia su actividad

Beneficia su actividad

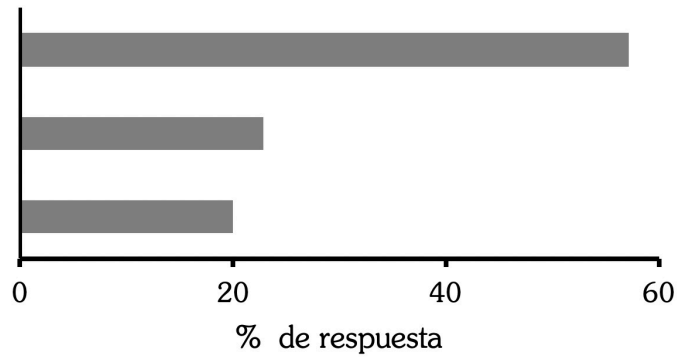

Fig. 2. Respuestas obtenidas por los pescadores artesanales de la región de Magallanes en cuanto al alcance de su percepción sobre qué es un área protegida (A) y qué tipo de efectos tiene sobre sus actividades (B).

MU fue que será una zona sin restricciones para el desarrollo de sus actividades, como así tampoco para las de otros actores, como por ejemplo el sector turístico (Fig. 3). Un $11 \%$ de los entrevistados manifestó que podrían darse restricciones al uso a partir de la creación del AMCP-MU, mientras que un $21 \%$ no tuvo ninguna apreciación con respecto al proyecto (Fig. 3).
Percepción sobre otros actores vinculados con la conservación de la biodiversidad de Magallanes

Con respecto a las instituciones vinculadas con la conservación y manejo de la biodiversidad marina y las áreas protegidas, se pudo observar que un $54 \%$ de los entrevistados percibían con cierta aprensión a los servicios públicos regionales (Fig. 4), principalmente debido a su rol fiscalizador

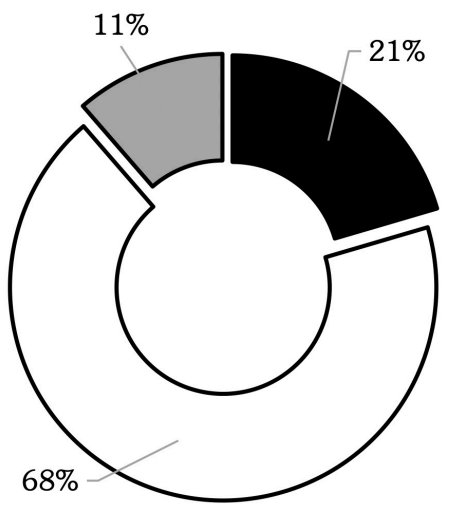

No tiene ninguna opinión aparente

- La percibe como una zona sin restricción para su actividad o para otro grupo de actores

口Percibe una futura AMCP como zona con restricciones sobre su actividad o para otro grupo de actores

Fig. 3. Percepción de los pescadores artesanales de la región de Magallanes frente a la posible instalación de un AMCP-MU en el Seno Almirantazgo. 
y de establecer reglamentaciones. Por su parte, las organizaciones no gubernamentales (ONGs) fueron mayormente percibidas en forma neutra (63\%) y sólo en un caso se registró una respuesta desfavorable sobre ellas (Fig. 4). El sector del turismo fue visualizado más favorablemente, pues un $57 \%$ de los entrevistados mostró percepciones positivas hacia el mismo (Fig.4); mientras que las asociaciones y comunidades indígenas fueron las que recibieron un mayor número de opiniones neutras (34 de los 35 entrevistados).

\section{DISCUSIÓN}

El éxito de acciones de conservación de la naturaleza está generalmente determinado por factores sociales, pues depende de procesos humanos en la toma de decisión y requieren de cambios en el comportamiento de las personas (Mascia et al. 2003). Por lo tanto, los estudios sociales contribuyen a identificar creencias, valores, normas y reglas que complementan la visión de las ciencias biológicas en el diseño y la implementación de acciones de conservación (Mascia et al. 2003). Más aun, debido a la interdependencia existente entre los recursos que proveen los ecosistemas y sus usuarios, la implementación efectiva de acciones de manejo ecosistémico en ambientes marinos depende de la identificación y comprensión de los actores involucrados con estos, sus prácticas, expectativas e intereses (Pomeroy \& Douvere, 2008). En este contexto, los resultados de este trabajo contribuyen al entendimiento de la dimensión humana la conservación marina en la región de Magallanes, atendiendo a las percepciones de los pescadores en relación a la biodiversidad marina, las áreas protegidas, el proyecto de creación de un AMCP-MU en el Seno Almirantazgo y los actores vinculados con la conservación de la biodiversidad en la región.

Conocimientos sobre conservación de la biodiversidad y áreas protegidas

Los entrevistados de nuestro estudio mostraron conocimientos sobre varias especies protegidas, las normas de los sitios que las protegen y la relación existente entre turismo - conservación. Esto último les permitió percibir uno de los servicios ecosistémicos clave de la región (Cáceres et al. 2015), tal como también ha sido visualizado en un estudio realizado por Cárcamo et al. (2014) en el norte de Chile.

Los pescadores entrevistados también demostraron tener conocimientos sobre las áreas protegidas de Magallanes. Si bien tenían en claro su ubicación y los límites aproximados, el nombre de las mismas no siempre resultó ser conocido. Por ejemplo, el Parque Marino Francisco Coloane fue más identificado a través de la isla Carlos III que por su nombre. A pesar de que esta AMP fue la primera en decretarse en Chile y que el proceso de creación implicó instancias participativas y de difusión (Programa de las Naciones Unidas para

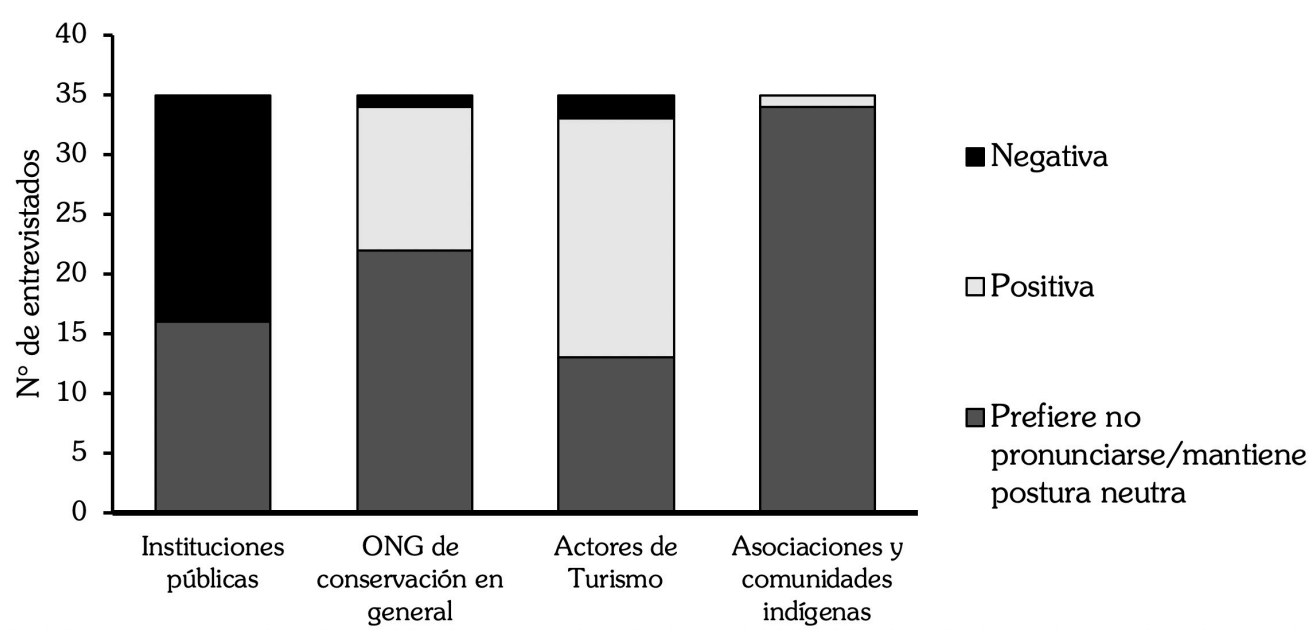

Fig. 4. Percepción de los pescadores artesanales de la región de Magallanes en relación a los actores sociales vinculados con la conservación y el manejo de la biodiversidad marina y las AMP. 
el Desarrollo, 2005; 2006; Ministerio del Medio Ambiente, 2018), los pescadores artesanales de Magallanes siguen reconociéndola más por un nombre ligado a la toponimia que por su nombre formal.

Entre las percepciones observadas se destaca la visión de que la conservación está orientada a la protección de especies y áreas frente a amenazas antrópicas, a desarrollar acciones ligadas al manejo de los recursos pesqueros o asociadas con la actividad turística. Algo semejante fue identificado por Cárcamo et al. (2014) en el norte de Chile, donde diversos actores sociales, entre ellos pescadores artesanales, visualizaron las AMP como una herramienta de conservación para la protección de la biodiversidad, los recursos pesqueros y la pesquería artesanal. Por su parte, Said (2016) concluyó que las AMP de Magallanes son valoradas por los pescadores por su contribución a la provisión de beneficios sociales, económicos y culturales. En particular, nuestros resultados indican que los pescadores perciben una asociación entre la protección de especies, las áreas protegidas y el turismo.

El concepto de área protegida tuvo un significado especial en nuestro estudio, pues los entrevistados la percibieron como una zona cerrada en la que tanto el acceso como las actividades humanas están prohibidos. Esto muestra la importancia y la influencia del vocabulario en las percepciones y como este puede incidir en las interacciones entre grupos de actores. Consecuentemente, parte de los entrevistados perciben a las áreas protegidas como una amenaza para su actividad, tal como ha sido observado en estudios donde los pescadores son los actores que muestran un menor grado de apoyo a la creación e implementación de AMP, por el temor a ver restringido su modo de vida e ingresos (Sunam et al. 1999; McClanahan et al. 2005; Jones, 2008; Kenneth \& Sanchirico, 2008; Charles \& Wilson, 2009; Hoelting et al. 2013).

Un estudio reciente sobre percepción de los servicios ecosistémicos y beneficios de la conservación marina en la AMCP-MU Seno Almirantazgo (Brain et al. 2020), indica que los principales beneficiarios de esta AMP serían los operadores turísticos, mientras que los pescadores habrían sido los menos beneficiados. A diferencia de algunas evaluaciones de percepción realizadas en otros sitios que han puesto de manifiesto un buen nivel de aceptación de las AMP, por los beneficios que aportan a las comunidades locales (Thomassin et al. 2010; Leleu et al. 2012; Lopes et al. 2013; Cárcamo et al. 2014; Callum et al. 2017), sólo un $20 \%$ de los entrevistados en nuestro estudio perciben estos beneficios y para la pesca en particular.

\section{Percepciones del proyecto de AMCP-MU en el Seno Almirantazgo}

A partir de la comprensión del alcance de un AMCP-MU, los pescadores no percibieron ninguna influencia negativa sobre su actividad y no mostraron una opinión negativa en relación a la creación de una AMP en el Seno Almirantazgo. Estudios complementarios desarrollados antes de la creación de esta AMP, basados en un enfoque de antropología aplicada al desarrollo sostenible (Agrech, 2015) o en la disposición a conservar (Said, 2016), arrojaron resultados semejantes en cuanto a esta percepción.

Esto podría explicarse sobre la base de las experiencias previas de los entrevistados, dado que no se encontraron con restricciones para trabajar en áreas protegidas, a semejanza de lo observado por Zorondo-Rodríguez et al. (2019). Por otra parte, entre los antecedentes históricos del área se destaca el colapso de la pesquería de ostión para el Seno Almirantazgo (Guzmán et al. 2010), como así también la solicitud de implementar medidas de protección del recurso y el sitio por el mismo sector de la pesca artesanal (S. Cornejo com. pers.). Estos antecedentes, además de que los pescadores no han visualizado restricciones asociadas a su actividad desde la creación del AMC-MU Francisco Coloane, podrían estar influenciado su percepción sobre las AMP.

Los entrevistados percibieron posibles consecuencias del AMCP-MU sobre la pesca, tanto negativas como positivas. En particular, visualizaron posibles molestias y amenazas asociadas al aumento de lobos marinos (Oliva et al. 2004) o beneficios asociados a la protección de los recursos pesqueros, tal como ha sido observado en otros estudios (Leleu et al. 2012; Lopes et al. 2013). Asimismo, manifestaron preocupación frente a 
posibles restricciones o la prohibición de acceso al área, la limitación de la cantidad de embarcaciones autorizadas a operar en la zona o las cuotas de extracción. Estas preocupaciones también fueron identificadas por Said (2016), como así también por Brain et al. (2020) después de haber sido creada el AMP. En este último estudio, el servicio ecosistémico priorizado por los pescadores fue la provisión de alimentos $\mathrm{y}$, consecuentemente, la mayoría de los encuestados visualizaron barreras potenciales en cuanto a posibles límites al derecho de extracción de recursos y restricciones de acceso al área.

\section{Percepción sobre otros actores}

La percepción sobre otros actores $u$ actividades podría explicar algunas dificultades y tensiones en relación a la futura creación e implementación de un AMP (Christie et al. 2003; Pomeroy et al. 2007a; Charles \& Wilson, 2009; Zorondo-Rodríguez et al. 2019; Pollnac et al. 2001, 2010). En nuestro estudio se pudo observar que los entrevistados mostraron cierta desconfianza frente a las autoridades. En particular, destacaron que, siendo los primeros interesados, en general, son dejados de lado para la toma de decisiones y que estas son tomadas a nivel central, por personas que desconocen las particularidades locales. Investigaciones realizadas sobre este mismo sector hacen también alusión a que las normas no son ajustadas a la realidad local, puesto que no se consideran las diferencias en los métodos y las faenas de pesca, los recursos y las condiciones geográficas y climáticas locales (WCS, 2019). En relación al sector académico, también se visualizan ciertas tensiones en términos de que, si bien este cuenta con conocimientos teóricos y empíricos sobre los recursos pesqueros, los entrevistados consideran que no tienen en cuenta su amplio conocimiento práctico y de terreno.

Es importante destacar que la pesca artesanal y el turismo comparten un mismo espacio de trabajo $y$ parecen no percibirse mutuamente en forma negativa. Estos dos sectores hacen uso del Seno Almirantazgo y el AMCP-MU y Parque Marino Francisco Coloane, e incluso algunos pescadores han comenzado a brindar servicios turísticos. Sin embargo, teniendo en cuenta el aumento de la visitación y el interés creciente por desarrollar actividades turísticas en el Seno Almirantazgo (Cáceres et al. 2015; Nahuelhual et al. 2019), la relación entre pescadores y operadores turísticos podría entrar en una situación particularmente instable, según cómo evolucionen sus actividades en el AMP.

\section{CONCLUSIONES}

Nuestro estudio pone en manifiesto la importancia de levantar información sobre los principales usuarios de las áreas en donde se considera crear AMP, previo a su declaratoria, de manera de construir una propuesta, planificación e implementación acorde con la visión, las necesidades y la percepción de estos actores, para potenciar el éxito del cumplimiento de los objetivos de conservación propuestos, tal como ha sido sugerido en otros estudios realizados para el Seno Almirantazgo (Agrech, 2015; Said, 2016; Brain et al. 2020) y a nivel nacional (ZorondoRodríguez et al. 2019; Cárcamo et al. 2014) e internacional (Christie et al. 2003; Pomeroy et al. 2007a; Pollnac et al. 2010; Charles \& Wilson, 2009; Heck \& Dearden, 2012). Por lo tanto, se recomienda generar espacios participativos e inclusivos para abordar la creación, la gobernanza y la gestión de las AMP de la región.

Los pescadores entrevistados en este estudio no se sintieron implicados en el proyecto del AMP en el Seno Almirantazgo, lo cual podría influenciar su percepción con relación a los costos y/o beneficios potenciales del mismo para su actividad. En ese sentido, un enfoque basado en la dimensión humana de la conservación permite visualizar el importante rol que juegan los usuarios de los sitios a proteger en las iniciativas de conservación. Más aun considerando que varios de los entrevistados en nuestro estudio se mostraron dispuestos a participar en la protección de las especies del área y en resolver los problemas relacionados con la basura que se presentan en el AMP. Este interés fue posteriormente canalizado en diferentes iniciativas participativas vinculadas con el diseño del AMP, la elaboración de su plan de manejo y el diagnóstico para la gestión de los residuos del área (Vila et al. 2017; Ministerio del Medio Ambiente 2018; WCS \& CMCC, 2019). 
Si bien las relaciones con las autoridades pesqueras locales no se ven como conflictivas, se mencionaron algunos puntos de tensión en relación a las actividades de control y fiscalización o de recolección, uso y presentación de datos por parte de instituciones atingente a estas acciones. En ese sentido, los encuentros en talleres $y$ Comités de Manejo pareciera que no se perciben como espacios que involucran a los pescadores en la toma de decisiones o que aún no se acostumbra a participar en ellos (Agrech, 2015). Por lo tanto, se deben movilizar acciones tanto para sociabilizar la relevancia como para motivar la participación en estos y otros espacios de trabajo conjunto en la búsqueda de la sustentabilidad social, económica y ambiental de las actividades que se desarrollan en los ecosistemas marinos.

\section{AGRADECIMIENTOS}

Agradecemos muy especialmente a cada uno de los pescadores artesanales que nos recibió y participó generosamente con su tiempo de las entrevistas, compartiendo un momento de sus labores diarias y sus conocimientos sobre los recursos marinos y la conservación. Agradecemos al equipo profesional de la Subsecretaría de Pesca y Acuicultura de la región de Magallanes, que revisó una versión preliminar del manuscrito y realizó recomendaciones que contribuyeron a mejorarlo. Asimismo, queremos reconocer el importante apoyo recibido por parte de Anaïs Agrech, en el marco del desarrollo de una práctica profesional, y Joselyn Said para la realización de las entrevistas junto a nuestro equipo de trabajo; como así también a Nykol Jara Reyes por la elaboración de la cartografía y The David \& Lucile Packard Foundation por financiar parcialmente este estudio. Finalmente, agradecemos a los tres evaluadores del manuscrito, dado que sus comentarios y sugerencias contribuyeron a mejorarlo sustancialmente.

\section{LITERATURA CITADA}

Aguayo - Lobo, A., Acevedo J., \& Olave, C. (2007). Actualización de las bases para una estrategia para la conservación de mamíferos marinos en la región de Magallanes y Antártica Chilena.
Chile. Fundación Centro de Estudios del Cuaternario (CEQUA), Fuego-Patagonia y Antártica, Punta Arenas, Chile.

Agrech, A. (2015). Complexite des representations, enjeux et Strategies d'acteurs autour d'un projet d'aire Marine protegee sur le fjord Almirantazgo en Patagonie chilienne. Tesis de Master "Anthropologie \& métiers du développement durable". Francia: Département d'anthropologie, Université Aix Marseille.

Bennett, J., \& Dearden, P. (2014). Why local people do not support conservation: Community perceptions of marine protected area livelihood impacts, governance and management in Thailand. Marine Policy, 44, 107-116.

Brain, M. J., Nahuelhual, L., Gelcich, S., \& Bozzeda, F. (2020). Marine conservation may not deliver ecosystem services and benefits to all: Insights from Chilean Patagonia. Ecosystem Services, 45, 101-170.

Cáceres B., Kush A., \& Vila, A. (2015). Manual de Buenas Prácticas para el turismo de intereses especiales en ecosistemas marinos y costeros australes. Punta Arenas, Chile. Wildlife Conservation Society-Chile.

Cárcamo, P., Garay-Flühmann, R., \& Gaymer, C. (2014). Collaboration and knowledge networks in coastal resources management: How critical stakeholders interact for multipleuse marine protected area implementation. Ocean \& Coastal Management, 91(1), 5-16.

Callum, R., Bethan, C., Douglas, J., Philippe, M., Duarte, C., Lubchenco J., Daniel, P., Saenz-Arroyo, A., Ussif Rashid S., Rod, W., Worm, B., \& Castilla, J. (2017). Marine reserves can mitigate and promote adaptation to climate change. Proceedings of the National Academy of Sciences, 114(24), 6167-6175.

Charles, A. \& Wilson, L. (2009). Human Dimensions of Marine Protected Areas. Ices Journal of Marine Science - ICES J MAR SCI, 66, 6-15.

Convention on Biological Diversity. (2010). Strategic Plan for Biodiversity 20112020, Convention on Biological Diversity. 
Montreal.

Christie, P., Mccay, B., Miller, M., Lowe, C., White, A., Stoffle, R., Fluharty, D., McManus, L., Chuenpagdee, R., \& Pomeroy, R. (2003). Toward developing a complete understanding: A social science research agenda for marine protected areas. Fisheries, 28, 22-25.

Di Minim E., Brook, T., Toivonen, T., Butchart, S., Heikinheimo, V., Watson, J., Burgess, N., Challender, D., Goettsch, B., Jenkins, R., \& Moilanen, A. (2019). Identifying global centers of unsustainable commercial harvesting of species. Science Advances, 5, 28-79.

Dudley, N. (2008). Guidelines for Applying Protected Area Management Categories. International Union for Conservation of Nature - IUCN.

Edgar, G., Stuart-Smith, D., Willis, J., Kininmonth, S., Baker, C., \& Banks, S. (2007). Partnership for Interdisciplinary Studies of Coastal Oceans (PISCO). The Science of Marine Reserves, 2, 1-22.

Falabella, V., Campagna, C., \& Croxall, J. (2009). Atlas del Mar Patagónico: Especies y Espacios. Wildlife Conservation Society and BirdLife International, Buenos Aires, Argentina.

Fernández, M., \& Castilla, J. C. (2005). Marine conservation in Chile: historical perspective, lessons, and challenges. Conservation Biology, 19(6), 1752 - 1762.

Fernández, M. P. (2014) Rastreando huellas, la dinámica del paisaje en el valle de la Paciencia, Tierra del Fuego. Tesis para optar al grado de Licenciatura en Antropología. Santiago Chile. Universidad Academia de Humanismo Cristiano.

Guzmán, L., Daza, E., Vargas, C., Leal, E., Vargas, C., Castillo, V., Bazán, V., Bucarey, D., \& Lara, E. (2010). Evaluación de bancos de ostión del sur (Chlamys vitrea) en seno de Almirantazgo y canal Beagle, Región de Magallanes y Antártica Chilena. Informe Final Corregido. FIP N²008-28. 144 pp.

Häussermann, V., \& Försterra, G. (2009). Marine benthic fauna of Chilean Patagonia: illustrated identification guide. Nature in
Focus: Santiago de Chile.

Heck, N., \& Dearden, P. (2012). Local expectations for future marine protected area performance: A case study of the proposed national marine conservation area in the southern strait of Georgia, Canada. Coastal Management, 40, 577-6593.

Hoelting, K. R., Hard, C. H., Christie, P., \& Pollnac, R. B. (2013). Factors affecting support for Puget Sound Marine Protected Areas. Fisheries Research, 144, 48-59.

Hucke-Gaete R., Lo Moro, P., \& Ruiz, J. (2010). Conservando el mar de Chiloé, Palena y Guaitecas: Síntesis del estudio Investigación para el desarrollo de Área Marina Costera Protegida Chiloé, Palena y Guaitecas. Editado por Gobierno Regional de Los Lagos, Chile.

Iriarte, J. L., González, H.E., \& Nahuelhual, L. (2010). Patagonian fjord ecosystems in southern Chile as a highly vulnerable region: problems and needs. AMBIO, 39, 463-466.

Jones, P. J. S. (2008). Fishing industry and related perspectives on the issues raised by no-take marine protected area proposals. Marine Policy, 32, 749-758.

Kenneth, J., \& Sanchirico, N. (2008). Local perspectives on marine reserve creation in the Bahamas. Ocean \& Coastal Management, 51, 763-771.

Kuempel, C., Jones, K., Watson, J., \& Possingham, H. (2019). Quantifying biases in marine-protected-area placement relative to abatable threats. Conservation Biology. $10 \mathrm{pp}$.

Kroodsma, D., Mayorga, J., Hochberg, T., Miller, N., Boerder, K., Ferretti, F., Wilson, A., Bergman, B., White, T., Block, B., Woods, P., Sullivan, B., Costello, C., \& Worm, B. (2018). Tracking the global footprint of fisheries. Science, 359, 904-908.

Leleu, K., Alban, F., Pelletier, D., Charbonnel, E., Letourneur, Y., \& Boudouresque, C. (2012). Fishers' perceptions as indicators of the performance of Marine Protected Areas. Marine Policy, 36, 414-422.

Lester, S., Halpern, S., Grorud-Colvert, K., Lubchenco, J., Ruttenberg, B., Gaines, D., \& Warner, R. (2009). Biological effects 
within no-take marine reserves: A global synthesis. Marine Ecology Progress Series, 384(1), 33-46.

Lopes, P. F. M., Rosa, E. M., Salyvonchyk, S., Nora, V., \& Begossi, A. (2013). Suggestions for fixing top-down coastal fisheries management through participatory approaches. Marine Policy, 40(1), 100110.

Mascia, M., Brosius, J., Dobson, T., Forbes, B., Horowitz, L, McKean, M., \& Turner, N. (2003). Conservation and the social sciences. Conservation Biology, 17(1), 649 - 650.

McCauley D. J., Pinsky, M. L., Palumbi, S. R., Estes, J. A., Joyce, F. H., \& Warner, R. (2015). Marine defaunation: Animal loss in the global ocean. Science, 347(6219), 1255641-1255641.

McClanahan, T., Davies, J., \& Maina, J. (2005). Factors influencing resource users and managers' perceptions towards marine protected area management in Kenya. Environmental Conservation, 32 (1), 42-49.

Ministerio del Medio Ambiente. (2018). Área Marina Costera Protegida de Múltiples Usos y Parque Marino Francisco Coloane: Plan Estratégico para el manejo de conservación y desarrollo de actividades sostenibles 2018-2027. Secretaría Regional Ministerial del Medio Ambiente de Magallanes y Antártica Chilena, Comité Operativo del Área Marina Costera Protegida de Múltiples Usos Francisco Coloane y Wildlife Conservación Society.

Ministerio del Medio Ambiente (2020) Registro Nacional de Áreas Protegidas. Ministerio del Medio Ambiente. http://areasprotegidas. mma.gob.cl/ Consultado en 28/04/2020.

Montiel, A., \& Jara, N. (2020). Contribución de las áreas marinas protegidas a la conservación del patrimonio natural de la XII región. Anales del Instituto de la Patagonia, Editorial, 48(1), 2.

Nahuelhual, L., Vergara, X., Jullian, C., Carmona, A., \& Campos, G. (2019). Servicios ecosistémicos marino-costeros en la Región de Magallanes y la Antártica Chilena. Centro de Investigación: Dinámica de Ecosistemas Marinos de Altas Latitudes, IDEAL.

Outeiro, L., Häussermann, V., Viddi, F., HuckeGaete, R., Försterra, G., Oyarzo, H., Kosiel, K., \& Villasante, S. (2015). Using ecosystem services mapping for marine spatial planning in southern Chile under scenario assessment. Ecosystem Services, 16, 341-353.

Oliva, D., Sielfeld, W., Durán, L., Sepúlveda, M., Pérez, M., Rodríguez, L., Stotz W., \& Araos, $\mathrm{V}$. (2004). Interferencia de mamíferos marinos con actividades pesqueras y de acuicultura. Informe final FIP N²003-32.

Palumbi, S., Sandifer, J., Allan, M., Beck, D., Fautin, M., Fogarty, S., Halpern, L., JoAnn Leong, I., Norse, E., Stachowicz, J., \& Wall, D. (2009). Managing for ocean biodiversity to sustain marine ecosystem services. Frontiers in Ecology and the Environment, 7(4), 204-211.

Pantoja, S., Iriarte, J.L., \& Daneri, G. (2011). Oceanography of the Chilean Patagonia. Continental Shelf Research, 31(3-4), 149153.

Poloczanska E. S., Burrows M. T, Brown C. J., García Molinos J., Halpern B. S., HoeghGuldberg O., Kappel C. V., Moore P. J., Richardson A. J., Schoeman D. S., \& Sydeman W. J. (2016). Responses of Marine Organisms to Climate Change across Oceans. Frontiers in Marine Science, 3, 62-??.

Pollnac, R., Christie, P., Cinner, J., Dalton, T., Daw, T., Forrester, G., Graham, N. \& Mcclanahan, T. (2010). Marine reserves as linked social-ecological systems. Proceedings of the National Academy of Sciences of the United States of America, 107, 18262-5.

Pomeroy, R., Parks, J., Pollnac, R., Campson, T., Genio, E., Marlessy, C., Holle, E., Pido, M., Nissapa, A., Boromthanarat, S. \& Thu Hue, N. (2007a). Fish wars: Conflict and collaboration in fisheries management in Southeast Asia. Marine Policy, 31, 645656.

Pomeroy, R., Mascia, M., \& Pollnac, R. (2007b). Marine protected areas, the social 
dimension. In FAO Expert workshop on marine protected areas and fisheries management: Review of issues and considerations, FAO Rome, 149-275.

Pomeroy, R., \& Douvere, F. (2008). The engagement of stakeholders in the marine spatial planning process. Marine Policy, 32, 816-822.

Programa de las Naciones Unidas para el Desarrollo. (2005). Plan general de administración área marino costera protegida de múltiples usos Francisco Coloane. Comité Regional AMCP-MU y Proyecto GEF.

Programa de las Naciones Unidas para el Desarrollo. (2006). Conservación de la biodiversidad de importancia mundial a lo largo de la costa chilena Áreas marinas y costeras protegidas de múltiples usos Isla Grande de Atacama, Lafken Mapu Lahual y Francisco Coloane. Ocho libros (Eds). Proyecto GEF y Gobierno de Chile.

Said, J. (2016). Disposición a conservar: una evaluación ex-ante de un Área Marina Protegida en Magallanes, Chile. Tesis de pregrado. Santiago, Chile: Facultad de Ciencias Universidad de Chile.

Servicio Nacional de Pesca y Acuicultura. (2019). Anuario Estadístico de Pesca. Consultado en http://www.sernapesca.cl/informacionutilidad/anuarios-estadisticos-de-pesca-yacuicultura.

Subsecretaría de Pesca y Acuicultura. (2010). Informe Técnico (R . Pesq ) N $138 / 2010$

Suspensión transitoria de la inscripción en el registro pesquero artesanal para los recursos ostión del sur Chlamys vitrea y ostión patagónico Chlamys patagonica en las Regiones de Los Lagos, Aysén y de Magallanes.

Sunam, D., Shivlani, M., \& Milon, J. (1999). Perceptions and attitudes regarding marine reserves: a comparison of stakeholder groups in the Florida Keys National Marine Sanctuary. Ocean \& Coastal Management, 42, 1019-1040.

Thomassin, A., White, C., Stead, S., \& David, G. (2010). Social acceptability of a marine protected area: the case of Reunion Island. Ocean \& Coastal Management, 53, 169179.
Edgar, G. J., Stuart-Smith, R. D., Willis, T. J., Kininmonth, S., Baker, S. C., Banks, S., ... \& Buxton, C. D. (2014). Global conservation outcomes depend on marine protected areas with five key features. Nature, 506(7487), 216-220.

Townsend, D., Hunt, R., McMullen, J., \& Sarasvathy, S. (2018). Uncertainty, knowledge problems, and entrepreneurial action. Academy of Management Annals, 12, 659-687.

UNEP-WCMC \& IUCN. (2020). Marine Protected Planet, Cambridge, UK: UNEP-WCMC and IUCN. Retrieved from www.protectedplanet. net

Unión Internacional para la Conservación de la Naturaleza. (2016). Resoluciones, recomendaciones y otras decisiones de la UICN. Gland, Suiza: UICN.

United Nations (2015). Transforming Our World: The 2030 Agenda for Sustainable Development (UNGA Resolution A/ RES/70/1, 25 September 2015) ('2030 Agenda').

Vila, A., Püschel, N., Rodríguez, M., Guijón, R., \& Kusch, A. (2017). Propuesta Área Marina Costera Protegida de Múltiples Usos Seno Almirantazgo. Tierra del Fuego, Región de Magallanes y de la Antártica Chilena. Informe técnico. Wildlife Conservation Society - Chile. Santiago.

WCS \& CMCC. (2019). Diagnóstico sobre la gestión actual de residuos en la pesca artesanal de la región de Magallanes y de la Antártica Chilena. Wildlife Conservation Society y Comité de Manejo de los recursos Centolla y Centollón de la región de Magallanes.

Worm B., Barbier, E., Beaumont, N., Duffy, J., Folke, C., Halpern, B., Jackson, J., Lotze, H., Micheli, F., Palumbi, S., Sala, E., Selkoe, K., Stachowicz, J., \& Watson, R. (2006). Impacts of Biodiversity Loss on Ocean Ecosystem Services. Science, 314, 787-79.

Worm B., Barbier, E., Beaumont, N., Duffy, J., Folke, C., Halpern, B., Jackson, J., Lotze, H., Micheli, F., Palumbi, S., Sala, E., Selkoe, K., Stachowicz, J., \& Watson, R. 
(2007). Response to Comments on Impacts of Biodiversity Loss on Ocean Ecosystem Services. Science, 316, 1285d.

Zorondo-Rodríguez F., Díaz, M., Simonetti-Grez, G., \& Simonetti, J. (2019). Why would new protected areas be accepted or rejected by the public?: Lessons from an ex-ante evaluation of the new Patagonia Park Network in Chile. Land Use Policy, 89, 104-248. 